\title{
Depressão Materna e a Interação Triádica Pai-Mãe-Bebê
}

\author{
Maternal Depression and Father-Mother-Baby Triadic Interaction
}

\author{
Giana Bitencourt Frizzo* \& Cesar Augusto Piccinini \\ Universidade Federal do Rio Grande do Sul, Porto Alegre, Brasil
}

\begin{abstract}
Resumo
O presente estudo examinou as eventuais diferenças na interação triádica (pai-mãe-bebê) e diádica (mãe-bebê, pai-bebê e mãe-pai) em famílias com e sem depressão materna, com bebês de um ano de idade, durante uma sessão de interação livre. Participaram do estudo 19 famílias, das quais 9 de mães deprimidas e 10 de mães não-deprimidas. Foram investigados os padrões de interação triádico e diádico através de um protocolo envolvendo diversas categorias. Contrariando a hipótese do estudo, não houve diferenças estatisticamente significantes nas interações triádicas entre as famílias com e sem depressão materna. Já nas interações diádicas, dentro de cada grupo de famílias, apareceram diferenças estatisticamente significantes no grupo sem depressão materna. Nas famílias com depressão materna, apenas a categoria estimulação cognitiva obteve significantemente maior incidência, indicando que, embora deprimidas, as mães conseguiam prover uma estimulação adequada para seus bebês. No conjunto, os dados sugerem que a depressão materna pode acarretar mudanças, ainda que sutis, no padrão familiar.

Palavras-chave: Depressão materna; interação triádica; interação familiar.
\end{abstract}

\begin{abstract}
This study examined the eventual differences in triadic (father-mother-baby) and dyadic (mother-baby, fatherbaby and father-mother) interaction in families with and without maternal depression, with one-year old babies, during a free-play session. Nineteen families participated in the study, 9 with maternal depression and 10 without. The triadic and the dyadic patterns of interaction were examined using a protocol with several categories. Contradicting the hypothesis of the study, there were no significant differences in the interactions among families with and without maternal depression. But when analyzing the dyadic interactions, in each group of families, there were some significant differences only in the group without maternal depression. In families with maternal depression, only the cognitive stimulation category was significantly more intense, indicating that despite the depression, the mother could provide adequate stimulation for their babies. On the whole, the results suggest that maternal depression may cause changes, even subtle, in the family pattern of interaction

Keywords: Maternal depression; triadic interaction; family interaction.
\end{abstract}

Diversos autores sugerem que, por vezes, a depressão materna pode surgir em algum outro momento do primeiro ano de vida do bebê e não necessariamente nas primeiras semanas após o seu nascimento, embora ainda fortemente associados à maternidade (Brown, Lumley, Small \& Astbury, 1994; Murray, Cox, Chapman \& Jones, 1995). Quando a depressão da mãe ocorre durante as quatro semanas após o parto, esta é classificada pelo DSM-IV, como episódio de depressão maior com início no pós-parto. Para a CID-10, a depressão pós-parto pode surgir durante as seis primeiras semanas após o parto.

Quando os sintomas de depressão da mãe ocorrem após este período e/ou se estendem ao longo do primeiro ano

* Endereço para correspondência: Universidade Federal do Rio Grande do Sul, Instituto de Psicologia, Rua Ramiro Barcelos, 2600, sala 111, Porto Alegre, RS, 90035-006. E-mail: gifrizzo@hotmail.com

Este artigo é baseado em parte da dissertação de mestrado de Giana Bitencourt Frizzo, realizada e apresentada no Curso de Pós-Graduação em Psicologia do Desenvolvimento da Universidade Federal do Rio Grande do Sul sob a supervisão de Cesar Augusto Piccinini. de vida do bebê, ela é por vezes denominada apenas de depressão materna (Coyl, Roggman \& Newland, 2002; Goodman \& Gotlib, 1999), embora outros autores ainda a denominem de depressão pós-parto (Chabrol, Bron \& Le Camus, 1996; Clark, Tluczek \& Wenzel, 2003; Coutinho, Baptista \& Morais, 2002; Cramer, 1997; Crockenberg \& Leerkes, 2003; Murray \& Cooper, 1997; Murray, Fioricowley, Hooper \& Cooper, 1996; Trad, 1997). Tendo em vista que o final do primeiro ano de vida do bebê foi o período investigado no presente estudo, o termo depressão materna foi escolhido. É importante também deixar claro que neste estudo a depressão materna foi avaliada pelo Inventário Beck de Depressão (Cunha, 2001) que dá apenas indicadores de depressão. Assim, o termo depressão materna, foi usado no presente estudo para denominar as mães que apresentaram indicadores de depressão no final do primeiro ano de vida do filho(a).

A depressão materna ocorre em torno de $10 \%$ das puérperas, conforme dados de literatura internacional (Cooper \& Murray, 1995; Dunnewold, 1997; O'Hara, Neunaber \& Zekoski, 1984). No entanto, estudos brasilei- 
ros têm apontado prevalências mais altas de depressão materna. No estudo de Cruz, Simões e Faisal-Cury (2005) $37 \%$ das puérperas em uma população de baixa renda, atendida pelo Programa de Saúde da Família, apresentaram critérios diagnósticos para depressão. Outro estudo brasileiro, realizado um mês após o parto, também encontrou uma prevalência mais alta de depressão, de $24 \%$, em puérperas (Souza \& Daure, 1999). Já no estudo de Coutinho, Baptista e Morais (2002), realizado entre três e seis meses após o parto, a prevalência encontrada foi em $32 \%$ das mulheres. Ainda que as amostras dos estudos acima não sejam representativas, e que tenham utilizado diferentes períodos após o parto para avaliação da depressão, seus resultados podem ser um indicativo de que a depressão materna pode ser mais freqüente em amostras brasileiras do que se preconizava com base na literatura internacional (Cooper \& Murray, 1995; Dunnewold, 1997; O'Hara et al., 1984). Talvez isso possa ser parcialmente explicado devido à associação entre a depressão materna e as dificuldades socioeconômicas da família (Robila \& Krishnakumar, 2005), uma vez que esses estudos enfocaram mulheres de classes populares, onde são freqüentes também estressores crônicos, tais como problemas de saúde da mãe, dificuldade com o bebê, fal ta de dinheiro para a satisfação de necessidades básicas, conflitos freqüentes com a rede de apoio, além de falta de apoio social, tanto em nível de informação quanto emocional (Seguin, Potvin, St-Denis \& Loiselle, 1999).

Caracteristicamente, a depressão materna compreende alterações no apetite e sono, dificuldade para dormir, especialmente após amamentar o bebê, crises de choro, desatenção, problemas de concentração, falta de energia e de interesse em atividades que antes eram consideradas agradáveis (Dunnewold, 1997). Também podem ocorrer idéias de suicídio e sentimentos excessivos de culpa. Os sintomas tendem a ter uma duração razoável de tempo e podem prejudicar as atividades normais da mulher (O'Hara, 1997), ainda que, de modo geral, a literatura indique que a depressão materna ao longo do primeiro ano de vida do bebê tende a ser leve (O'Hara et al., 1984; Steiner \& Tam, 1997; Whiffen \& Gotlib, 1989). De acordo com Steiner e Tam (1997), o fato de a depressão ser geralmente de intensidade leve ou moderada, faz com que freqüentemente ela não seja diagnosticada (Frizzo \& Piccinini, 2005; Schwengber \& Piccinini, 2003), podendo eventualmente tornar-se tão severa que uma internação seja necessária. Para Maldonado (2000), a severidade da depressão da mãe parece estar relacionada com uma grande frustração das expectativas relacionadas à maternidade, ao seu papel materno, ao bebê e ao tipo de vida que é estabelecido com a chegada da criança.

\section{Depressão Materna e a Interação Mãe-Bebê e Pai-Bebê}

A literatura tem sido consistente em caracterizar que mães deprimidas tendem a ser menos responsivas a seu bebê do que as não deprimidas (Brazelton \& Cramer, 1992; Campbell, Cohn, \& Meyers, 1995; Field, 2000; Field, Healy, Goldstein \& Guthertz, 1990; Field et al., 1988; Frizzo \&
Piccinini, 2005; Hoffman \& Drotar, 1991; Murray et al. 1996; Schwengber, 2002; Schwengber \& Piccinini, 2003; Stern, 1997; Teti \& Gelfand, 1991). A depressão tenderia a afetar a disponibilidade cognitiva e emocional da mãe, condições inerentes à contingência das respostas, por isso ela pode não conseguir ser adequadamente responsiva à sua criança. De acordo com Teti e Gelfand (1991), como as mães deprimidas também tendem a ficar mais reclusas e ensimesmadas, pode ocorrer uma inatenção e insensibilidade à saúde, à segurança e às necessidades psicológicas de sua criança (Teti \& Gelfand, 1997; Teti, Gelfand, Messinger \& Isabella, 1995). Além disso, de acordo com Cohn, Campbell, Mattias e Hopkins (1990), a falta de sensibilidade materna nos primeiros meses de vida parece provocar uma mudança evolutiva no bebê, pois esta falta de sensibilidade tem sido empiricamente relacionada a um baixo envolvimento com pessoas e objetos ao final do primeiro ano de vida do bebê. Tal mudança pode ter implicações importantes para a criança, mesmo quando há remissão da patologia materna.

Os bebês são muito vulneráveis ao impacto da depressão materna porque dependem muito da qualidade dos cuidados e da sensibilidade da mãe (Campbell et al., 1995). Nos casos em que o pai está presente, seu papel passa a ter grande relevância para a família, uma vez que a sua participação como mediador da interação mãe-bebê pode ter uma influência direta sobre o desenvolvimento da criança (Brazelton \& Cramer, 1992).

Além disso, muitos autores referem que os pais são normalmente tão competentes quanto as mães na interação com seus filhos e filhas pequenos, por isso, é possível que uma interação positiva pai-bebê possa compensar parcialmente uma interação mãe-bebê negativa ou insuficientemente boa (Belsky, Gilstrap \& Rovine, 1984; Chabrol et al., 1996, Hops et al., 1987; Parke, 1996). Os poucos estudos que consideraram o papel do pai frente à depressão materna mostraram que este pode exercer um papel protetor para o desenvolvimento infantil nessas situações (Hops et al., 1987; Hossain et al., 1994). Ele pode prover um modelo positivo, tanto aumentando os cuidados com seus filhos, quanto apoiando a mãe deprimida, o que contribui para uma melhor criação dos filhos.

No estudo de Hossain et al. (1994) foram examinadas as interações mãe-bebê e pai-bebê em situação de depressão materna. Foram investigadas 26 famílias americanas com bebês entre três e seis meses, das quais doze mães estavam deprimidas. Cada díade interagiu por três minutos durante uma situação de interação face-a-face. Os resultados sugeriram que o pai pode amenizar os efeitos negativos da depressão materna para o bebê, pois os pais das famílias cujas mães estavam deprimidas apresentaram mais expressões faciais positivas e vocalizações do que as mães deprimidas e também os bebês mostraram mais expressões faciais positivas e vocalizações ao interagirem com seus pais. Tais resultados divergem de outros estudos (Field et al., 1988).

No estudo de Field et al. (1988), foi investigada a interação face-a-face em 74 díades mãe-bebê, com crian- 
ças de 3 a 6 meses de idade. Primeiro houve um período de interação com a mãe deprimida e posteriormente com uma mulher adulta não deprimida, que o bebê não conhecia. Os resultados desse estudo indicaram que houve um efeito de generalização do padrão deprimido de interação da interação mãe-bebê para a situação estranha-bebê, pois não apenas o bebê apresentou o mesmo padrão de interação de quando com sua mãe, como acabou eliciando na estranha um comportamento mais apático. Já no estudo de Hossain et al. (1994) não ocorreu essa generalização do padrão deprimido de interação face-a-face do bebê com sua mãe deprimida para a situação com seu pai, que lhe era familiar e sensível às suas necessidades, além não estar deprimido. Possivelmente essa diferença deva-se ao fato de que no estudo de Field et al. (1988) foi analisada a interação do bebê com uma pessoa estranha, o que pode vir a ser mais estressante para o bebê do que interagir com seu pai, como ocorreu no estudo de Hossain et al., ou com uma pessoa que não lhe seja estranha, como a atendente da creche usada no estudo de Pelaez-Nogueras, Field, Cigales, Gonzalez e Clasky (1995). Neste último também não houve generalização do padrão deprimido de interação dos bebês de mães deprimidas durante a interação com a atendente de creche.

Apesar da importância do estudo da família como unidade de análise poucos estudos investigaram as relações familiares em situação de depressão materna. Por exemplo, no estudo feito por Hops et al. (1987), os pais das famílias nas quais a mãe estava deprimida eram mais envolvidos com seus filhos e filhas do que as mães, mas apenas quando havia um bom relacionamento conjugal, indicando que quando a mãe está deprimida, o pai tenderia a compensar as falhas da parceira. Além disso, as mães deprimidas evidenciaram maiores taxas de afeto disfórico, isto é, tristeza e desânimo, e menores índices de afeto alegre nas interações com o marido e com os filhos, sugerindo que mulheres deprimidas comportam-se diferentemente de mulheres saudáveis em seus lares.

\section{Método}

\section{Participantes}

Participaram deste estudo dezenove famílias, 9 das quais com mães deprimidas e 10 com mães sem depressão, de acordo com os escores da mãe no Inventário Beck de Depressão (BDI - Beck \& Steer, 1993). Os bebês eram saudáveis, nascidos a termo e tinham, aproximadamente, um ano de idade. Todas as mães eram primíparas e residiam com seus maridos ou companheiros. Nenhuma delas relatou complicações durante a gestação. A idade média das mães foi de 24 anos $(D P=4,75)$ e dos pais 27 anos $(D P=8,22)$.

As famílias selecionadas para o presente estudo faziam parte do "Estudo Longitudinal de Porto Alegre: Da gestação à Escola", que vem acompanhando, aproximadamente, 100 famílias de diferentes idades, configurações familiares e níveis socioeconômicos, desde a gestação do primeiro filho até o quarto ano de vida da criança. Os participantes foram selecionados após preencherem a Ficha de Contato Inicial (Grupo de Interação Social, Desenvolvimento e
Psicopatologia [GIDEP], 1998a). Os casais que aceitaram participar do estudo e preencheram os critérios exigidos (ex: estar esperando o primeiro filho, estar no terceiro trimestre da gestação, não apresentar complicações físicas durante a gestação) foram contatados posteriormente por telefone.

Para fins do presente estudo, foram inicialmente selecionadas todas as 47 famílias cuja mãe havia respondido o Inventário Beck de Depressão, quando o bebê estava com um ano de idade. Das mães que foram avaliadas pelo BDI, 30 não apresentavam indicadores de depressão $(63,8 \%$; BDI $\mathrm{d} \leq 11), 11$ apresentavam indicadores de depressão leve $(23,4 \%$; BDIe $\geq 12)$ e 6 apresentavam indicadores de depressão moderada $(12,8 \%$; BDI $\geq 20)$. Foi oferecido atendimento psicoterápico por psicólogas integrantes do projeto longitudinal para as mães que apresentaram depressão.

Dentre as mães que responderam ao $\mathrm{BDI}, 19$ foram selecionadas para fins de emparelhamento quanto ao nível socioeconômico da família (Hollingshead, 1975) e ao sexo do bebê. O teste Qui-quadrado não revelou diferenças estatisticamente significantes quanto aos dados sócio-demográficos nas famílias com e sem depressão materna.

\section{Delineamento e Procedimentos}

Foi utilizado um delineamento de grupos contrastantes (Nachmias \& Nachmias, 1996), envolvendo dois grupos: um de famílias com depressão materna e outro de famílias sem depressão materna. Em cada grupo foram examinadas as interações triádicas (pai-mãe-bebê) e diádicas (mãebebê, pai-bebê e mãe-pai).

Quando o bebê completou um ano de idade, as famílias foram convidadas a comparecer à Sala de Brinquedos do Instituto de Psicologia da Universidade Federal do Rio Grande do Sul (UFRGS) para a filmagem da Observação da interação familiar (GIDEP, 1998a). Após, a mãe preencheu o Inventário Beck de Depressão. Nesta ocasião também foram utilizados outros instrumentos de avaliação que não serão considerados no presente estudo ${ }^{1}$. O Consentimento Informado (GIDEP, 1998b) já havia sido assinado na primeira fase de coleta de dados do projeto longitudinal e abrangia também esta fase de coleta de dados.

\section{Instrumentos e Materiais}

1. Ficha de Contato Inicial (GIDEP, 1998a): esse instrumento investigou alguns dados demográficos, tais como idade da mãe e do companheiro, escolaridade, profissão, estado civil, e existência de outros filhos.

2.Inventário Beck de Depressão (Beck \& Steer, 1993; Cunha, 2001): essa escala foi utilizada para avaliar a depressão materna. O BDI é uma escala sintomática de auto-relato, composta por 21 itens com diferentes alternativas de resposta a respeito de como o sujeito tem se sentido, e que correspondem a diferentes

${ }^{1}$ Também foi aplicada a Entrevista sobre o desenvolvimento do bebê e a experiência da maternidade (GIDEP, 2000b) e a Entrevista sobre o desenvolvimento do bebê e a experiência da paternidade (GIDEP, 2000c). 
níveis de gravidade da depressão. A versão em português do BDI resultou de uma formulação consensual da tradução do original em inglês, com a colaboração de quatro psicólogos clínicos, quatro psiquiatras e uma tradutora, sendo testada junto com a versão em inglês em 32 pessoas bilíngües, com três dias de intervalo e variando a ordem da apresentação dos dois idiomas nas duas metades da amostra (Cunha, 2001; Cunha, Prieb, Goulart \& Lemes, 1996). A consistência interna do BDI foi de 0,84 e a correlação entre teste e reteste foi de $0,95(p<0,001)$. A soma dos escores dos itens individuais fornece um escore total, que por sua vez constitui um escore dimensional da intensidade da depressão, que pode ser classificado nos seguintes níveis: mínimo (até 11 pontos), leve (de 12 a 19 pontos), moderado (de 20 a 35 pontos) ou grave (acima de 36 pontos).

3. Observação da Interação Familiar (GIDEP, 2000a): a filmagem da sessão de observação foi composta de quatro episódios: pai-mãe-bebê, mãe-bebê, pai-bebê e novamente pai-mãe-bebê. Cada episódio interativo durou, aproximadamente, oito minutos. Os pais foram orientados a agir da maneira mais natural possível, como faziam quotidianamente com a criança.

A avaliação da interação familiar foi realizada de acordo com a Avaliação da interação triádica e diádica (Núcleo de Estudos em Infância e Família [NUDIF], 2003), baseado em Cox (1998). Para fins deste estudo, foram avaliados apenas os seis minutos finais de cada um dos dois episódios de interação triádica pai-mãe-bebê. Este período de tempo foi dividido em 12 intervalos de um minuto cada, durante os quais se buscou avaliar diversas categorias de interação triádica. O protocolo utilizado foi composto de sete categorias: sensibilidade, afeto positivo, afeto negativo, desengajamento, intrusividade, estimulação cognitiva e alianças familiares. (a) Sensibilidade: refere-se à sensibilidade dos pais às necessidades, ao humor, aos interesses e às capacidades da criança; (b) Afeto positivo: refere-se à extensão na qual os membros da família parecem apreciar estar juntos; eles são carinhosos entre si e parecem relaxados e à vontade uns com os outros; (c) Afeto negativo: refere-se às expressões de afeto negativo, conflitos, discordâncias ou críticas de um membro da família em relação a outro; (d) Desengajamento: reflete a extensão na qual os membros da família parecem não engajados, emocionalmente não envolvidos, não responsivos e não conscientes das necessidades uns dos outros; (e) Intrusividade: refere-se às interações intrusivas e supercontroladas, centradas no adulto e não na criança; (f) Estimulação cognitiva: refere-se às tentativas de estimular o desenvolvimento cognitivo e mental da criança; (g) Alianças familiares: refere-se às interações entre dois membros da família no sentido de excluir um terceiro membro. Conforme sugestão de Cox (1998), dentro de cada intervalo de tempo, a análise das interações triádicas buscou investigar a qualidade da interação entre todos os membros da família, mesmo quando tais intenções foram dirigidas a um membro da família. Após a avaliação geral da interação triádica, os intervalos de tempo foram novamente examinados, quando se assinalou a presença das categorias de análise para cada uma das interações diádicas (i.e. mãebebê, pai-bebê e mãe-pai). Dois codificadores, cegos para o diagnóstico de depressão materna, procederam à análise dos vídeos, após, aproximadamente, 25 horas de treinamento com outras fitas de vídeo não utilizadas para este estudo. Após esse treinamento inicial, seis casos foram selecionados para o cálculo do índice de concordância, realizado pelo Teste Kendall (SPSS, 1997). Esse índice de concordância foi calculado para cada categoria e contexto interativo e variou de 0,79 (categoria estimulação cognitiva mãe-bebê) a 1,0O (categoria desengajamento mãe-bebê). A média do índice de concordância foi 0,88. Em algumas categorias, devido à falta de variabilidade nos escores, não foi possível fazer o cálculo pelo Teste de Kendall, então foi necessário realizar uma análise de concordância simples, que variou entre 0,67 (categoria intrusividade triádica) e 1,OO (categoria aliança familiar triádica, aliança familiar mãe-bebê, aliança familiar pai-bebê, aliança familiar mãepai) $(M=0,89 ; D P=0,13)$. Depois de estabelecidos todos os índices de concordância, os dois codificadores examinaram todos os demais vídeos, sendo que eventuais diferenças foram dirimidas, com o auxílio de um terceiro codificador, no caso, a pesquisadora.

\section{Resultados}

O teste não-paramétrico Mann-Whitney, com opção exata, foi realizado para cada categoria examinada nos contextos interativos triádico e diádico. A Tabela 1 apresenta a média, o desvio padrão e o nível de significância para cada categoria e contexto interativo para as famílias com e sem depressão materna. A análise não revelou nenhuma diferença estatisticamente significante entre os escores das famílias com e sem depressão materna, tanto para as interações triádicas como diádicas. Apenas algumas tendências não significantes aparecerem em algumas categorias. $\mathrm{Na}$ categoria afeto positivo, no contexto interativo mãe-pai, houve uma incidência maior de respostas $(p<0,13)$ nas famílias com depressão materna $(M=11,33)$ do que em famílias sem depressão materna $(M=6,90)$. Tendência semelhante apareceu na categoria afeto negativo $(p<0,12)$, também no contexto interativo mãe-pai, que apresentou maior incidência nas famílias com depressão materna $(M=4,89)$ do que em famílias sem depressão materna $(M=2,50)$. Por fim, na categoria desengajamento, no contexto interativo mãe-bebê, as díades mãe-bebê das famílias com depressão materna $(M=16,33)$ tenderam a apresentar mais desengajamento $(p<0,10)$ do que as díades das famílias sem depressão materna $(M=13,10)$. De qualquer maneira, essas diferenças não significantes e as análises não apóiam a hipótese inicial de que haveria diferenças nos diferentes contextos interativos triádico e diádico entre as famílias com e sem depressão materna. 
Frizzo, G. B. \& Piccinini, C. A. (2007). Depressão Materna e a Interação Triádica Pai-Mãe-Bebê.

Tabela 1

Incidência Média, Desvio Padrão e Nível de Significância por Categoria e Contexto Interativo nas Famílias com e sem Depressão

\begin{tabular}{|c|c|c|c|c|}
\hline Categoria & $\begin{array}{l}\text { Contexto } \\
\text { interativo }\end{array}$ & $\begin{array}{l}\text { Com depressão } \\
\qquad(n=9)\end{array}$ & $\begin{array}{l}\text { Sem depressão } \\
(n=10)\end{array}$ & $p<$ \\
\hline \multirow[t]{3}{*}{ Sensibilidade } & Triádica & $48,22(8,74)$ & $49,30 \quad(6,53)$ & 0,47 \\
\hline & Mãe-bebê & $50,11 \quad(11,38)$ & $52,70 \quad(6,18)$ & 0,62 \\
\hline & Pai-bebê & $43,33 \quad(12,83)$ & $44,40 \quad(10,55)$ & 0,96 \\
\hline \multirow[t]{4}{*}{ Afeto positivo } & Triádico & $45,56 \quad(10,47)$ & $46,30 \quad(8,31)$ & 0,72 \\
\hline & Mãe-bebê & $47,33 \quad(14,03)$ & $49,60 \quad(7,05)$ & 0,98 \\
\hline & Pai-bebê & $39,33 \quad(16,12)$ & $39,00 \quad(13,46)$ & 0,99 \\
\hline & Mãe-pai & $11,33(8,48)$ & $6,90(7,11)$ & 0,13 \\
\hline \multirow[t]{4}{*}{ Afeto negativo } & Triádico & $12,56(1,33)$ & $12,00 \quad(0,00)$ & 0,47 \\
\hline & Mãe-bebê & $12,00(0,00)$ & $12,00 \quad(0,00)$ & 1,00 \\
\hline & Pai-bebê & $12,00(0,00)$ & $11,90 \quad(0,32)$ & 1,00 \\
\hline & Mãe-pai & $4,89(4,05)$ & $2,50 \quad(2,68)$ & 0,12 \\
\hline \multirow[t]{3}{*}{ Desengajamento } & Triádico & $18,22(4,20)$ & $16,20 \quad(4,69)$ & 0,31 \\
\hline & Mãe-bebê & $16,33(7,47)$ & $13,10(3,48)$ & 0,10 \\
\hline & Pai-bebê & $20,11(8,67)$ & $19,00 \quad(8,90)$ & 0,80 \\
\hline \multirow[t]{3}{*}{ Intrusividade } & Triádico & $12,78 \quad(1,09)$ & $13,30 \quad(1,34)$ & 0,64 \\
\hline & Mãe-bebê & $12,78(1,30)$ & $12,80 \quad(1,62)$ & 0,59 \\
\hline & Pai-bebê & $12,33(0,70)$ & $13,00(1,25)$ & 0,13 \\
\hline \multirow[t]{3}{*}{ Estimulação Cognitiva } & Triádico & $15,22(2,22)$ & $16,80(5,39)$ & 0,69 \\
\hline & Mãe-bebê & $15,78(2,54)$ & $17,30 \quad(6,99)$ & 0,89 \\
\hline & Pai-bebê & $13,00 \quad(1,32)$ & $14,20(3,73)$ & 1,00 \\
\hline \multirow[t]{4}{*}{ Aliança familiar } & Triádico & $13,89(1,69)$ & $14,00 \quad(2,36)$ & 1,00 \\
\hline & Mãe-bebê & $12,56(2,55)$ & $13,40 \quad(2,80)$ & 0,45 \\
\hline & Pai-bebê & $11,22(2,17)$ & $10,40 \quad(2,50)$ & 0,42 \\
\hline & Mãe-pai & $10,33(1,50)$ & $10,20(2,35)$ & 0,90 \\
\hline
\end{tabular}

A Tabela 2 apresenta a incidência média, o desvio padrão e o nível de significância para cada categoria para os contextos interativos mãe-bebê e pai-bebê nas famílias com e sem depressão materna.

Tabela 2

Incidência Média, Desvio Padrão e Nível de Significância das Categorias e Contextos Interativos Mãe-bebêe Pai-bebê

\begin{tabular}{|c|c|c|c|c|c|c|}
\hline & \multicolumn{3}{|c|}{ Com depressão $\quad(n=9)$} & \multicolumn{3}{|c|}{ Sem depressão $(n=10)$} \\
\hline & Mãe-bebê & Pai-bebê & $p<$ & Mãe-bebê & Pai-bebê & $p<$ \\
\hline Sensibilidade & $50,11 \quad(11,38)$ & $43,33 \quad(12,83)$ & 0,19 & $52,70 \quad(6,18)$ & $44,40 \quad(10,55)$ & 0,06 \\
\hline Afeto positivo & $47,33(14,03)$ & $39,33(16,12)$ & 0,26 & $49,60 \quad(7,05)$ & $39,00 \quad(13,46)$ & $\mathrm{O}, \mathrm{O} 3$ \\
\hline Afeto negativo & $12,00(0.00)$ & $12,00(\mathrm{O}, \mathrm{OO})$ & $1, \mathrm{OO}$ & $12,00(\mathrm{O}, \mathrm{OO})$ & $11,90(0,32)$ & $1, \mathrm{OO}$ \\
\hline Desengajamento & $16,33(7,47)$ & $20,11 \quad(8,67)$ & 0,38 & $13,10 \quad(3,48)$ & $19,00(8,90)$ & 0,09 \\
\hline Intrusividade & $12,78 \quad(1,30)$ & $12,33(\mathrm{O}, 7 \mathrm{O})$ & 0,51 & $12,80 \quad(1,62)$ & $13,00(1,25)$ & 0,80 \\
\hline Estimulação cognitiva & $15,78 \quad(2,54)$ & $13, \mathrm{OO}(1,32)$ & 0,03 & $17,30 \quad(6,99)$ & $14,20 \quad(3,73)$ & 0,01 \\
\hline Aliança familiar & $12,56(2,55)$ & $11,22(2,17)$ & 0,48 & $13,40 \quad(2,80)$ & $10,40(2,50)$ & 0,19 \\
\hline
\end{tabular}

O teste não paramétrico Wilcoxon, com opção exata, revelou poucas diferenças significantes nas diversas análises realizadas. Dentre elas, destaca-se a categoria afeto positivo $(p<0,03)$ que apareceu com mais intensidade no contexto interativo mãe-bebê do que pai-bebê nas famílias sem depressão materna. Já a categoria estimulação cognitiva foi mais intensa no contexto interativo mãe-bebê do que pai-bebê, tanto nas famílias com depressão materna $(p<0,03)$ como nas famílias sem depressão materna $(p<0,01)$. Por fim, na categoria sensibilidade foi revelado um efeito marginalmente significativo $(p<0,06)$, indicando maior intensidade dessa categoria no contexto interativo mãe-bebê do que pai-bebê nas famílias sem depressão materna. Outro efeito marginalmente significativo $(p<0,09)$ apareceu na categoria desengajamento, indicando uma maior incidência no contexto interativo pai-bebê do que mãe-bebê nas famílias sem depressão materna. Apesar dessas diferenças, no conjunto, os dados não corroboram 
as hipóteses iniciais do presente estudo que indicavam diferenças significantes, particularmente nas famílias com depressão materna.

\section{Discussão}

Os resultados do presente estudo não corroboraram as hipóteses iniciais, sobre diferenças nas interações entre famílias com mães deprimidas e sem depressão, derivadas de vários estudos examinados. Contudo, os resultados aproximam-se daqueles do estudo conduzido por Chabrol et al. (1996) que investigou a interação face-a-face mãebebê e pai-bebê em contexto diádico e as díades mãe-bebê e pai-bebê em contexto triádico em vinte famílias com e sem depressão materna quando os bebês tinham cinco meses de idade. À semelhança dos dados do presente estudo, os autores também não encontraram diferenças estatisticamente significantes entre os grupos e revelaram que mães moderadamente deprimidas podiam inclusive apresentar interações positivas com seus filhos e filhas.

Encontrou-se uma possível explicação para tais resultados no estudo de Frankel e Harmon (1996), que investigou a relação entre a depressão materna em instrumentos de auto-relato e dados observacionais entre 30 mulheres deprimidas e 32 sem depressão, nos Estados Unidos. Os dados de observação não evidenciaram diferenças na interação das mães com seus filhos entre os grupos de mães com e sem depressão. No entanto, o auto-relato das mães deprimidas era mais negativo, pois todas mães deprimidas desse estudo se percebiam como mais tristes, relataram maior estresse com a maternidade, maior insatisfação conjugal e que tinham crianças menos adaptativas e mais estressantes. Contudo, os dados mostraram que nem sempre essas percepções se traduziram em interações disfuncionais entre a mãe e sua criança, talvez indicando que mães deprimidas parecem ser particularmente resilientes, pelo menos durante as interações com seus filhos, especialmente por se preocuparem com um possível impacto negativo da sua depressão sobre seus filhos, o que não significa que elas não estejam sofrendo com os sintomas depressivos.

Os resultados do presente estudo indicaram também uma tendência não significante tanto de maior afeto positivo quanto de afeto negativo nas díades mãe-pai em famílias com depressão materna, relativamente às famílias sem depressão materna. Embora no presente estudo não houvesse hipótese quanto a esse aspecto, as emoções são um aspecto importante quando se está lidando com transtornos afetivos (Phares, Duhig \& Watkins, 2002). A depressão materna pode estar relacionada o conflito conjugal (Cummings \& Davies, 1994; O’Hara, 1997; Romito, SaurelCubizolles \& Lelong, 1999) o que justificaria uma eventual expressão de afeto mais negativo entre o casal. Já Phares et al. (2002) relataram que mães deprimidas podem ser pouco coerentes nas suas demonstrações de afeto, o que poderia explicar parcialmente os resultados encontrados.

No presente estudo também buscou-se examinar as possíveis diferenças entre as díades mãe-bebê e pai-bebê em famílias com e sem depressão materna. Era esperado que nas díades pai-bebê de famílias com depressão materna houvesse maior expressão de afeto positivo e menor expressão de afeto negativo nas interações pai-bebê do que nas díades mãe-bebê (Hossain et al., 1994). Apenas a categoria estimulação cognitiva foi significantemente diferente entre as díades mãe-bebê e pai-bebê em famílias com depressão materna, indicando que, apesar da depressão, as mães estimularam mais seus bebês do que os pais. Já nas famílias sem depressão materna, as díades mãe-bebê apresentaram significantemente mais afeto positivo e mais estimulação cognitiva do que as díades pai-bebê. Houve ainda uma diferença marginalmente significante na mesma direção na categoria sensibilidade. Por fim, nessas famílias houve também maior desengajamento nas díades pai-bebê do que nas díades mãe-bebê. Isso não corrobora a hipótese inicial do estudo, que esperava maior expressão de afeto positivo e menor de afeto negativo nas interações pai-bebê do que mãe-bebê em famílias com depressão materna, em uma tentativa de o pai compensar os possíveis efeitos negativos da depressão materna para a criança (Hops et al., 1987; Hossain et al., 1994; Minuchin, 1982; Minuchin \& Fishman, 1990).

É possível que no presente estudo, o pai tenha apenas amenizado os eventuais efeitos da depressão materna para a família em contexto triádico, visto que a literatura tem relatado diferenças na interação mãe-bebê, quando esta ocorre em contexto diádico, ao se comparar famílias com e sem depressão (Campbell et al., 1995; Field, 2000; Field et al., 1990; Field et al., 1988; Hart, Field, del Valle \& Pelaez-Nogueras, 1998; Hoffman \& Drotar, 1991; Murray et al., 1996; Schwengber, 2002; Teti \& Gelfand, 1991). De acordo com Hops et al. (1987) parece haver uma interação complexa entre relacionamento conjugal e a interação paimãe-bebê, pois quando há satisfação conjugal, o cônjuge não deprimido conseguiria compensar as possíveis falhas na parentagem do cônjuge doente. $\mathrm{O}$ autor também sugeriu que talvez os padrões de interação estabelecidos na família sejam mais importantes do que o diagnóstico parental isoladamente.

Assim, embora no presente estudo não tenham ocorrido diferenças estatisticamente significantes entre as famílias com e sem depressão materna, é plausível supor que a depressão materna possa afetar o padrão familiar. Isso recebe pelo menos apoio parcial dos resultados do presente estudo, que mostraram que as interações mãe-bebê e paibebê pouco diferiram nas famílias com depressão materna, ao contrário do que apareceu nas famílias sem depressão. Nestas últimas, comparada à interação pai-bebê, a interação mãe-bebê tendeu a apresentar maior incidência de comportamentos na maioria das categorias examinadas: sensibilidade, afeto positivo, estimulação cognitiva e alianças familiares. Ou seja, parece que em famílias sem depressão materna há uma tendência de as díades mãebebê serem mais ativas do que as díades pai-bebê. Uma possível explicação para esse achado pode ser oferecida pela teoria sistêmica (Minuchin, 1982), ao afirmar que as mudanças numa estrutura familiar podem contribuir para 
mudanças no comportamento e nos processos psíquicos internos dos outros membros desse sistema, como parece ter ocorrido parcialmente nas famílias com depressão materna examinadas no presente estudo.

Um outro fator que pode ter contribuído para os achados do presente estudo é o próprio diagnóstico de depressão materna, que é um tanto controverso na literatura. Em uma revisão realizada por Lovejoy, Graczyk, O'Hara e Neuman (2000), os autores indicaram que instrumentos de auto-relato, como o Inventário Beck de Depressão pode não ser fidedigno aos critérios diagnósticos de depressão de entrevistas diagnósticas estruturadas. No entanto, grande parte dos estudos utiliza esse instrumento na avaliação da depressão (Chabrol et al., 1996; Cramer, 1997; Field, 1997; Field et al., 1988; Hart et al., 1998; Hops et al., 1987; Hossain et al., 1994; Kelley \& Jennings, 2003; Matthey, Barnett, Ungerer \& Waters, 2000; Pickens \& Field, 1993; Schwengber, 2002; Schwengber \& Piccinini, 2003; Seguin et al., 1999; Teti et al., 1995; Whiffen \& Gotlib, 1989). De qualquer forma, novos estudos poderiam utilizar também uma entrevista diagnóstica, a fim de confirmar o diagnóstico de depressão.

Já de acordo com Cummings e Davies (1994), um grande problema no diagnóstico da depressão é que ela muitas vezes parece ser considerada unidimensional. Para exemplificar isso, os autores explicaram que muitos estudos apenas dividem os grupos em deprimidos e não deprimidos, desconsiderando outros fatores que podem influenciar o diagnóstico de depressão. Novos estudos poderiam investigar longitudinalmente a influência da depressão materna para a família, pois depressões crônicas parecem ter um maior impacto nas relações familiares do que episódios de depressão transitórios (Campbell et al., 1995; Cohn et al., 1990; Field, 1997; Murray, 1992). Alguns autores indicam inclusive que parece haver um aumento das expressões de afeto negativo ao longo do tempo se a depressão persistir (Cohn et al., 1990). A severidade da depressão também poderia ser investigada em novos estudos, pois tem sido um fator pouco mencionado na literatura e que também poderia explicar os diferentes impactos da depressão da mãe na família. Além disso, de acordo com Minuchin (1982), também seria importante investigar como ocorre a readaptação dos papéis familiares quando há remissão da sintomatologia materna. De qualquer modo, a depressão pode aparecer associada a vários fatores que mesmo na ausência desta, podem fazer surgir padrões familiares disfuncionais, como baixo nível socioeconômico, pouco conhecimento sobre desenvolvimento infantil e eventos de vida estressantes (Campbell \& Cohn, 1997; Cummings \& Davies, 1994).

Um fator que também pode explicar a ausência de diferenças entre as famílias com e sem depressão materna é a presença do pai do bebê. Assim como no estudo de Campbell e Cohn (1997), as mães do presente estudo eram casadas e o companheiro estava presente e envolvido com a criança em algum nível. O próprio fato de o pai envolver-se, desde a gestação no projeto longitudinal, do qual o presente estudo faz parte, pode ser um indicador do envolvimento desses pais com seu filho e família. Conforme Phares et al. (2002), ter uma boa relação afetiva como uma das figuras parentais, neste caso o pai, pode ser um fator de grande proteção para a criança. Também é possível que as mães do presente estudo tenham recebido apoio suficiente do companheiro e da família, o que pode ter evitado que elas tivessem problemas na interação com seus bebês.

Possivelmente mais do que o tipo de análise utilizado, o tamanho pequeno da amostra acabou reduzindo o poder estatístico dos testes utilizados. Além disso, o contexto de interação triádico também pode ter contribuído para essa ausência de diferenças, uma vez que a presença do cônjuge claramente altera o contexto interativo entre a criança e o outro cônjuge, transformando a díade em um complexo sistema familiar que inclui os subsistemas conjugal e parental. É possível que a própria situação de interação livre não tenha permitido a expressão de intrusividade como ocorreria em situações de interação estruturada, pois não exige uma atitude mais efetiva da mãe e do pai durante a interação com a criança, conforme já apontado por Schwengber (2002) e Schwengber e Piccinini (2003).

Uma importante limitação do presente estudo foi que os pais não foram avaliados quanto à depressão. Inicialmente tentou-se fazer essa avaliação, mas como alguns pais recusaram-se a preencher o BDI desistiu-se de aplicá-lo aos demais. Sugere-se que esse fator seja investigado em estudos futuros, pois é possível que em famílias onde ambos os pais estejam deprimidos o contexto de interação com o filho e entre o casal seja particularmente difícil.

Finalmente, é importante salientar que, conforme a extensa revisão da literatura realizada por Cummings e Davies (1994), mesmo em ambientes familiares com muitas características depressivas, é possível que atuem alguns fatores de resiliência que possam amenizar os efeitos negativos da depressão. Embora a depressão esteja comumente associada à discórdia familiar, em muitas famílias com pais ou mães deprimidos, parece haver um funcionamento saudável no casamento, no manejo com as crianças e na provisão de afeto para estas. Soma-se a isso, segundo os autores, o fato de que a vivência nesse ambiente pode ter resultados positivos, pois algumas crianças com essa história familiar podem se tornar especialmente sensíveis aos sentimentos e às necessidades dos outros, o que pode ser valorizado e adaptativo em alguns contextos de suas vidas.

\section{Considerações Finais}

O presente estudo teve como objetivo examinar as interações triádicas e diádicas entre famílias com e sem depressão materna. Os resultados não corroboraram as hipóteses do estudo, contrariando boa parte da literatura sobre depressão materna. Nesse sentido, é importante que se tenha cautela na interpretação desses resultados e vários pontos levantados acima precisam ser considerados. De qualquer modo, ainda existem inconsistências na literatura, o que aponta para a necessidade de novos estudos, 
que corroborem ou não os achados do presente estudo. É possível que o pai exerça um papel moderador quanto aos eventuais efeitos da depressão materna para a família, talvez amenizando tais efeitos no contexto triádico, visto que parece haver uma alteração do padrão familiar nas famílias com depressão materna.

É importante ressaltar que a depressão materna constitui-se em um fenômeno bastante complexo, passível de inúmeras variações, seja na intensidade como na cronicidade. Assim sendo, é plausível supor-se que, como destacado nos dados do presente estudo, muitas díades mãebebê, bem como suas famílias, possam ter um funcionamento semelhante a famílias não afetadas pela depressão. As pesquisas deveriam também buscar compreender quais os fatores que estão mais comumente associados com a depressão materna, dentre os quais pode-se destacar conflitos conjugais, ausência de planejamento da gravidez, temperamento e sexo do bebê, relação com a família de origem, e eventos de vida estressantes que estejam ocorrendo no momento. Com tantos fatores potencialmente importantes para as interações familiares talvez o foco na depressão per se seja inadequado para explicar as variações nos padrões de triádicos e diádicos dentro da família. Além disso, estudar a depressão isoladamente, acaba desconsiderando o papel da criança e do seu ambiente no seu desenvolvimento.

Apesar das inconsistências, ainda existentes, quanto ao impacto da depressão materna para o desenvolvimento precoce, é importante que os profissionais da saúde estejam atentos para a qualidade da interação mãe-bebê, tendo em vista evidências relatadas na literatura sobre possíveis transtornos que a depressão materna pode gerar para a criança e também para a família. Dados epidemiológicos indicam que pelo menos $10 \%$ das puérperas desenvolvem depressão nos meses seguintes ao nascimento do bebê e, em várias delas, esse quadro poderá se estender ao longo de vários meses. Isso já se constitui razão suficiente para que se continue investigando a extensão do eventual impacto desse quadro, especialmente para o desenvolvimento do bebê. Nesse contexto, trabalhos preventivos junto a famílias de risco serão bem-vindos e contribuirão para que a depressão materna não tenha efeitos negativos no desenvolvimento infantil e familiar.

\section{Referências}

Beck, A. T., \& Steer, R. A. (1993). Beck Depression Inventory. Manual. San Antonio, CA: Psychological Corporation.

Belsky, J., Gilstrap, B., \& Rovine, M. (1984). The Pennsylvania infant and family development project, I: Stability and change in mother-infant and father-infant interaction in a family setting at one, three and nine months. Child Development, 55, 692-705.

Brazelton, T. B., \& Cramer, B. G. (1992). As primeiras relações (M. B. Cipolla, Trad.). São Paulo, SP: Martins Fontes.

Brown, S., Lumley, J., Small, R., \& Astbury, J. (1994). Missing. voices: The experience of motherhood. New York: Oxford University Press.
Campbell, S. B., \& Cohn, J. F. (1997). Chronicity of postpartum depression. In L. Murray \& P. J. Cooper (Eds.), Postpartum depression and child development (pp. 163-197). New York: The Guilford Press.

Campbell, S. B., Cohn, J. F., \& Meyers, T. (1995). Depression in first-time mothers: Mother-infant interaction and depression chronicity. Developmental Psychology, 31, 349-357.

Chabrol, H., Bron, N., \& Le Camus, J. (1996). Mother-infant and father-infant interactions in postpartum depression. Infant Behavior and Development, 19, 149-152.

Clark, R., Tluczek, A., \& Wenzel, A. (2003). Psychotherapy for postpartum depression: A preliminary report. American Journal of Orthopsychiatry, 73, 441-454.

Cohn, J. F, Campbell, S. B, Mattias, R., \& Hopkins, J. (1990). Face-to-face interactions of postpartum depressed and nondepressed mother-infant pairs at two months Developmental Psychology, 26, 15-23.

Cooper, P. J., \& Murray, L. (1995). Course and recurrence of postnatal depression: Evidence for the specificity of the diagnostic concept. British Journal of Psychiatry, 166, 191-195.

Coutinho, D. S., Baptista, M. N., \& Morais, P. R. (2002). Depressão pós-parto: Prevalência e correlação com o suporte social. Infanto - Revista de Neuropsiquiatria da Infância e Adolescência, 10, 63-71.

Cox, M. J. (1998). The young family interaction coding system. UNCCH, Chapel Hill. Manuscrito não-publicado.

Coyl, D. D., Roggman, L. A., \& Newland, L. A. (2002). Stress, maternal depression, and negative mother-infant interactions in relation to infant-attachment. Infant Mental Health, 23, 145163.

Cramer, B. G. (1997). Psychodynamic perspectives on the treatment of postpartum depression. In L. Murray \& P. J. Cooper (Eds.), Postpartum depression and child development (pp. 237-261). New York: The Guilford Press.

Crockenberg, S. C., \& Leekers, E. M. (2003). Parental acceptance, postpartum depression and maternal sensitivity: Mediating and moderating processes. Journal of Family Psychology, 17, 80-93.

Cruz, E. B. S., Simões, G. L., \& Faisal-Cury, A. (2005). Rastreamento da depressão pós-parto em mulheres atendidas pelo Programa de Saúde da Família. Revista Brasileira de Ginecologia e Obstetrícia, 27, 181-188.

Cummings, M. E., \& Davies, P. T. (1994). Maternal depression and child development. Journal of Child Psychology and Psychiatry, 35, 73-112

Cunha, J. A. (2001) Escalas Beck. São Paulo, SP: Casa do Psicólogo. Cunha, J. A., Prieb, R. G. G., Goulart, P. M., \& Lemes, R. B. (1996). O uso do inventário de Beck para avaliar depressão em universitários. Psico, 27(1), 107-115.

Dunnewold, A. L. (1997) Evaluation and treatment of postpartum emotional disorders. Sarasota, FL: Professional Resource Press.

Field, T. (1997) Depressed mothers and their infants. In L. Murray \& P. J. Cooper (Eds.), Postpartum depression and child development (pp. 22 1-236.). New York: The Guilford Press.

Field, T. (2000). Infants of depressed mothers. Stress, coping and depression (pp. 3-22). Mahwah, NJ: Lawrence Erlbaum.

Field, T., Healy, B., Goldstein, S., \& Guthertz, M. (1990). Behavior state-matching and sychrony in mother-infant interactions of non-depressed versus depressed dyads. Developmental Psychology, 26, 7-14

Field, T., Healy, B., Goldstein, S., Perry, S., Bendell, D., Shanberg, S., Zimmerman, E. A., \& Kuhn, C. (1988). Infants of depressed mothers show «depressed» behavior even with nondepressed adults. Child Development, 59, 1569-1579. 
Frankel, K. A., \& Harmon, R. J. (1996). Depressed mothers: They don't always look as bad as they feel. Journal of the American Academy of Child and Adolescent Psychiatry, 35, 289-298.

Frizzo, G. B., \& Piccinini, C. A. (2005). Interação mãe-bebê em contexto de depressão materna: Aspectos teóricos e empíricos. Psicologia em Estudo, 10, 47-55.

Goodman, S. H., \& Gotlib, I. H. (1999). Risk for psychopathology in the children of depressed mothers: A developmental model for understanding mechanisms of transmission. Psychological review, 106, 458-490.

Grupo de Interação Social, Desenvolvimento e Psicopatologia. (1998a). Ficha de contato inicial. Instituto de Psicologia, Universidade Federal do Rio Grande do Sul, Porto Alegre. Manuscrito não publicado.

Grupo de Interação Social, Desenvolvimento e Psicopatologia. (1998b). Consentimento informado. Instituto de Psicologia, Universidade Federal do Rio Grande do Sul, Porto Alegre. Manuscrito não publicado.

Grupo de Interação Social, Desenvolvimento e Psicopatologia. (2000a). Observação da interação familiar. Instituto de Psicologia, Universidade Federal do Rio Grande do Sul, Porto Alegre. Manuscrito não publicado.

Grupo de Interação Social, Desenvolvimento e Psicopatologia. (200ob). Entrevista sobre o desenvolvimento do bebê e a experiência da maternidade. Instituto de Psicologia, Universidade Federal do Rio Grande do Sul, Porto Alegre. Manuscrito não publicado.

Grupo de Interação Social, Desenvolvimento e Psicopatologia. (2000c). Entrevista sobre o desenvolvimento do bebê e a experiência da paternidade. Instituto de Psicologia, Universidade Federal do Rio Grande do Sul, Porto Alegre. Manuscrito não publicado.

Hart, S., Field, T., del Valle, C., \& Pelaez-Nogueras, M. (1998). Depressed mothers' interactions with their one-year-old infants. Infant Behavior and Development, 21, 519-525.

Hoffman, Y., \& Drotar, D. (1991). The impact of postpartum depressed mood on mother-infant interaction: Like mother, like baby? Infant Mental Health Journal, 12, 65-80.

Hollingshead, A. B. (1975). Four factor index of social status. Manuscrito não publicado.

Hops, H., Biglan, A., Sherman, L., Arthur, J., Friedman, L., \& Osteen, V. (1987). Home observations of family interactions of depressed women. Journal of Consulting and Clinical Psychology, 55, 341-346.

Hossain, Z., Field, T., Gonzalez, J., Malphurs, J., Del Valle, C., \& Pickens, J. (1994). Infants of «depressed» mothers interact better with their nondepressed fathers. Infant Mental Health Journal, 15, 348-357.

Lovejoy, M. C., Graczyk, P. A., O'Hara, E., \& Neuman, G. (2000). Maternal behavior and parenting behavior: a meta-analytic review. Clinical Psychology Review, 20, 561-592.

Maldonado, M. T. (2000). Psicologia da gravidez. São Paulo, SP: Saraiva.

Matthey, S., Barnett, B., Ungerer, J., \& Waters, B. (2000). Paternal and maternal depressed mood during the transition to parenthood. Journal of Affective Disorders, 60, 75-85.

Minuchin, S. (1982). Famílias: Funcionamento e tratamento. (J A. Cunha, Trad.). Porto Alegre, RS: Artes Médicas.

Minuchin, S., \& Fishman, H. C. (1990). Famílias. In Técnicas de terapia familiar (C. Kinsch \& M. E. R. F. Maia, Trad.). (pp. 2136). Porto Alegre, RS: Artes Médicas.

Murray, L. (1992). The impact of postnatal depression on infant development. Journal of Child Psychology and Psychiatry, 33, 543-561.
Murray, L., \& Cooper, P. J. (1997). Postpartum depression and child development. New York: The Guilford Press.

Murray, L., Cox, J., Chapman, G., \& Jones, P. (1995). Childbirth: Life event or start of a long-term difficulty? British Journal of Psychiatry, 166, 595-600.

Murray, L., Hooper, R., King, F., \& Fiori-Cowley, A. (1996). The role of infant factors in postnatal depression and motherinfant interactions. Developmental Medicine and Child Neurology, 38, 109-119

Nachmias, C., \& Nachmias, D. (1996) Research methods in the social sciences. London: Arnold.

Núcleo de Estudos em Infância e Família. (2003). Protocolo para avaliação da interação triádica e diádica. Instituto de Psicologia, Universidade Federal do Rio Grande do Sul, Porto Alegre. Manuscrito não publicado.

O'Hara, M. W. (1997). The nature of postpartum depressive disorders. In L. Murray \& P. J. Cooper. Postpartum depression and child development (pp. 3-34). New York: The Guilford Press.

O'Hara, M. W., Neunaber, D. J., \& Zekoski (1984). A prospective study of postpartum depression: Prevalence, course and predictive factors. Journal of Abnormal Psychology, 93, 158-171.

Parke, R. D. (1996). Fatherhood. London: Harvard University Press.

Pelaez-Nogueras, M., Field, T., Cigales, M., Gonzalez, A., \& Clasky, S. (1995). Infants of depressed mothers show less «depressed» behavior with their nursery teachers. Infant Mental Health Journal, 15, 358-367.

Phares, V., Duhig, A. M., \& Watkins, M. M. (2002). Family context: Fathers and other supports. In S. H. Goodman \& I. H. Gotlib. Children of depressed parents (pp. 203-226). Washington, DC: American Psychological Association.

Pickens, J., \& Field, T. (1993). Facial expressivity in infants of depressed mothers. Developmental Psychology, 29, 986-988.

Robila, M., \& Krishnakumar, A. (2005). Effects of economic pressure on marital conflict in Romania. Journal of Family Psychology, 19, 246-251

Romito, P., Saurel-Cubizolles, M., \& Lelong, N. (1999). What makes new mothers unhappy: Psychological distress one year after birth in Italy and France. Social Science \& Medicine, 49, 1651-1661.

Rutter, M. (1990). Commentary: Some focus and process considerations regarding effects of parental depression on children. Developmental Psychology, 26, 60-67.

Schwengber, D. D. S. (2002). A interação mãe-bebê e a experiência da maternidade de mães com e sem indicadores de depressão no final do primeiro ano de vida do bebê. Dissertação de Mestrado não-publicada, Curso de Pós-Graduação em Psicologia do Desenvolvimento, Universidade Federal do Rio Grande do Sul, Por to Alegre, RS

Schwengber, D. D. S., \& Piccinini, C. A. (2003). O impacto da depressão pós-parto para a interação mãe-bebê. Estudos de Psicologia-Campinas, 8, 403-411.

Seguin, L., Potvin, L., St-Denis, M., \& Loiselle, J. (1999). Depressive symptoms in the late postpartum among low socieconomic status women. Birth, 26(3), 157-163.

Souza, I., \& Daure, S. (1999). Importância da família na prevenção da depressão pós-parto. Psicologia Clínica, 11, 117-132.

Steiner, M., \& Tam, W. Y. K. (1997). Postpartum depression in relation to other psychiatric disorders. In L. J. Miller (Ed.), Postpartum mood disorders (pp. 47-64). Washington, DC: American Psychiatric Press.

Stern, D. (1997). A constelação da maternidade: O panorama da psicoterapia pais/bebêe (M. A. V. Veronese, Trad.). Porto Alegre, RS: Artes Médicas. 
Teti, D. M., \& Gelfand, D. M. (1991). Behavioral competence among mothers of infants in the first year: The mediational role of maternal self-efficacy. Child Development, 62, 918-929.

Teti, D. M., \& Gelfand, D. M. (1997) Maternal cognitions as mediators of child outcomes in the context of postpartum depression. In L. Murray \& P. J. Cooper (Eds.), Postpartum depression and child development (pp. 136-163). New York: The Guilford Press.

Teti, D. M., Gelfand, D., Messinger, D. S., \& Isabella, R. (1995). Maternal depression and the quality of early attachment: An examination of infants, preschoolers and their mothers. Developmental Psychology, 31, 364-376.

Trad, P. (1997). Psicoterapia breve pais/bebê (M. C. Müller \& M. E. Schestatsky, Trad.). Porto Alegre, RS: Artes Médicas.

Whiffen, V. E., \& Gotlib, I. H. (1989). Infants of postpartum depressed mothers: Temperament and cognitive status. Journal of Abnormal Psychology, 98, 274-279. 